Research Article

\title{
Notes on Local and Nonlocal Intuitionistic Fuzzy Fractional Boundary Value Problems with Caputo Fractional Derivatives
}

\author{
Ali El Mfadel (D), Said Melliani $(\mathbb{D}$, and M'hamed Elomari \\ Laboratory of Applied Mathematics and Scientific Computing, Sultan Moulay Slimane University, Beni Mellal 23000, Morocco \\ Correspondence should be addressed to Ali El Mfadel; elmfadelali@gmail.com
}

Received 25 April 2021; Accepted 10 August 2021; Published 24 August 2021

Academic Editor: Ram Jiwari

Copyright (C) 2021 Ali El Mfadel et al. This is an open access article distributed under the Creative Commons Attribution License, which permits unrestricted use, distribution, and reproduction in any medium, provided the original work is properly cited.

In this paper, we investigate the existence and uniqueness results of intuitionistic fuzzy local and nonlocal fractional boundary value problems by employing intuitionistic fuzzy fractional calculus and some fixed-point theorems. As an application, we conclude this manuscript by giving an example to illustrate the obtained results.

\section{Introduction}

Fuzzy fractional calculus has become a powerful tool with more accurate and successful results in modeling several complex and fuzzy physical phenomena in numerous seemingly diverse and widespread fields of science and engineering. Recently, the theory of fuzzy fractional differential equations was proposed to handle uncertainty due to incomplete information that appears in many mathematical or computer models of some deterministic real-world phenomena. When a real physical phenomenon is modeled by a fractional initial value problem, we cannot usually be sure that the model is perfect. For example, the initial value of this problem may not be known precisely. In order to get a perfect model under a precise initial condition, Agarwal et al., in [1], proposed the concept of fuzzy solutions for fractional differential equations with uncertainty. Arshad and Lupulescu, in [2], proved some results on the existence and uniqueness of solutions for fuzzy fractional differential equations. Later, Alikhani and Bahrami [3] proved the existence and uniqueness results for nonlinear fuzzy fractional integral and integrodifferential equations by using the method of upper and lower solutions. The authors in $[4,5]$ discussed the concepts about generalized Hukuhara fractional Riemann-Liouville and Caputo differentiability of fuzzy-valued functions. The equivalence between fuzzy fractional differential equation and fuzzy fractional integral equation was discussed in [6]. Ngo et al., in [7], proved the existence and uniqueness results of solutions for initial value problem under fuzzy Caputo-Katugampola fractional derivatives. For many basic works related to the nonlinear ordinary differential equations and the fuzzy fractional differential equations, we refer the readers to [8-13] and references therein.

Motivated by the results mentioned above and by using the intuitionistic fuzzy sets theory introduced by Atanassov, in [14], we study the existence and uniqueness results for the following intuitionistic fuzzy local and nonlocal fractional boundary value problems:

$$
\begin{aligned}
& \left\{\begin{array}{l}
{ }^{c} D^{\alpha} X(t)=F(t, X(t)), \quad t \in[0, T], \\
X(0)=a X(T),
\end{array}\right. \\
& \left\{\begin{array}{l}
{ }^{c} D^{\alpha} X(t)=F(t, X(t)), \quad t \in[0, T], \\
X(0)=G(X),
\end{array}\right.
\end{aligned}
$$

where ${ }^{c} D_{\alpha}$ is the Caputo derivative of $X(t)$ at order $0<\alpha<1$ and $F:[0, T] \times \mathbb{\mathbb { F } ^ { 1 }} \longrightarrow \mathbb{\mathbb { F } ^ { 1 }}$ and $G: C\left([0, T], \mathbb{\mathbb { F } ^ { 1 }}\right) \longrightarrow \mathbb{\mathbb { F } ^ { 1 }}$ are intuitionistic fuzzy continuous functions.

The spaces $\square \mathbb{F}^{1}$ and $C\left([0, T], \square \mathbb{F}^{1}\right)$ will be defined after.

Our paper is organized as follows. Section 2 gives some basic definitions, lemmas, and theorems as preliminaries of intuitionistic fuzzy sets theory. The existence results for the intuitionistic fuzzy local and nonlocal fractional boundary value problems are given in Section 3 and Section 4. Illustrative example is presented in Section 5, followed by conclusion and future works in Section 6. 


\section{Preliminaries}

Fuzzy set theory was introduced by Zadeh [15], and it is an extension of the classical crisp logic into a multivariate form. Atanassov generalizes this concept to intuitionistic fuzzy sets (IFSs) [14], and later, there has been much progress in the study of IFSs. As a special case of intuitionistic fuzzy sets, intuitionistic fuzzy numbers were introduced by $\mathrm{Xu}$ [16].

We denote by

$$
\mathbb{Q}(\mathbb{R})=\left\{\langle u, v\rangle: \mathbb{R} \longrightarrow[0,1]^{2}, 0 \leq u(x)+v(x) \leq 1\right\} .
$$

Definition 1 (see [17]). An element $\langle u, v\rangle \in \mathbb{F}(\mathbb{R})$ is called an intuitionistic fuzzy number if it satisfies the following conditions:

(1) $\langle u, v\rangle$ is normal, i.e., there exists $x_{0}, x_{1} \in \mathbb{R}$ such that $u\left(x_{0}\right)=1$ and $v\left(x_{1}\right)=1$

(2) $u$ is fuzzy convex and $v$ is fuzzy concave

(3) $u$ is upper semicontinuous and $v$ is lower semicontinuous

(4) $\operatorname{supp}\langle u, v\rangle=\{\overline{x \in \mathbb{R}: v(x)<1}\}$ is bounded

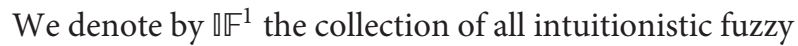
numbers.

Let $\alpha \in[0,1]$ and $\langle u, v\rangle \in \mathbb{\mathbb { F } ^ { 1 }}$, and we define the upper and lower $\alpha$-cuts of $\langle u, v\rangle$, respectively, by

$$
\begin{aligned}
& {[\langle u, v\rangle]^{\alpha}=\{x \in \mathbb{R}: v(x) \leq 1-\alpha\},} \\
& {[\langle u, v\rangle]_{\alpha}=\{x \in \mathbb{R}: u(x) \geq \alpha\} .}
\end{aligned}
$$

We define also the intuitionistic fuzzy zero denoted by $0_{\mathbb{V}} \in \mathbb{\mathbb { F } ^ { 1 }}$ as follows:

$$
0_{\mathbb{V F}}(t)= \begin{cases}(1,0) & \text { if } t=0 \\ (0,1) & \text { if } t \neq 0\end{cases}
$$

Definition 2 (see [17]). Let $\left\langle u_{1}, v_{1}\right\rangle,\left\langle u_{2}, v_{2}\right\rangle \in \mathbb{\mathbb { F } ^ { 1 }}, \lambda \in \mathbb{R}$, and $\alpha \in[0,1]$; then,

(1) $\left(\left\langle u_{1}, v_{1}\right\rangle \oplus\left\langle u_{2}, v_{2}\right\rangle\right)(z)=\left(\sup _{z=x+y} \min \left(u_{1}(x), u_{2}\right.\right.$ $\left.(y)), \inf _{z=x+y} \max \left(u_{1}(x), u_{2}(y)\right)\right)$

(2) $\lambda\left\langle u_{1}, v_{1}\right\rangle=\left\langle\lambda u_{1}, \lambda v_{1}\right\rangle$ if $\lambda \neq 0$

(3) $\lambda\left\langle u_{1}, v_{1}\right\rangle=0_{\text {IF }}$ if $\lambda=0$

(4) $\left[\left\langle u_{1}, v_{1}\right\rangle \oplus\left\langle u_{2}, v_{2}\right\rangle\right]^{\alpha}=\left[\left\langle u_{1}, v_{1}\right\rangle\right]^{\alpha}+\left[\left\langle u_{2}, v_{2}\right\rangle\right]^{\alpha}$

(5) $\left[\left\langle\left\langle u_{1}, v_{1}\right\rangle \oplus\left\langle u_{2}, v_{2}\right\rangle\right]_{\alpha}=\left[\left\langle u_{1}, v_{1}\right\rangle\right]_{\alpha}+\left[\left\langle u_{2}, v_{2}\right\rangle\right]_{\alpha}\right.$

(6) $\left[\lambda\left\langle u_{1}, v_{1}\right\rangle\right]^{\alpha}=\lambda\left[\left\langle u_{1}, v_{1}\right\rangle\right]^{\alpha}$

(7) $\left[\lambda\left\langle u_{1}, v_{1}\right\rangle\right]_{\alpha}=\lambda\left[\left\langle u_{1}, v_{1}\right\rangle\right]_{\alpha}$

Let $\langle u, v\rangle \in \mathbb{Q} \mathbb{F}^{1}$ and $\alpha \in[0,1]$; then, we define the following sets:

$$
\begin{aligned}
& {[\langle u, v\rangle]_{l}^{+}(\alpha)=\inf \{x \in \mathbb{R}: u(x) \geq \alpha\},} \\
& {[\langle u, v\rangle]_{r}^{+}(\alpha)=\sup \{x \in \mathbb{R}: u(x) \geq \alpha\},} \\
& {[\langle u, v\rangle]_{l}^{-}(\alpha)=\inf \{x \in \mathbb{R}: v(x) \leq 1-\alpha\},} \\
& {[\langle u, v\rangle]_{r}^{-}(\alpha)=\sup \{x \in \mathbb{R}: v(x) \leq 1-\alpha\} .}
\end{aligned}
$$

Remark 1. Let $\langle u, v\rangle \in \mathbb{Q} \mathbb{F}^{1}$ and $\alpha \in[0,1]$; then, we have

$$
\begin{aligned}
& {[\langle u, v\rangle]^{\alpha}=\left[[\langle u, v\rangle]_{l}^{-}(\alpha),[\langle u, v\rangle]_{r}^{-}(\alpha)\right],} \\
& {[\langle u, v\rangle]_{\alpha}=\left[[\langle u, v\rangle]_{l}^{+}(\alpha),[\langle u, v\rangle]_{r}^{+}(\alpha)\right] .}
\end{aligned}
$$

Definition 3 (see [17]). Let $\langle u, v\rangle \in \mathbb{E}^{1}$ and $\alpha \in[0,1]$, and we define the diameter of upper and lower $\alpha$ - cuts of $\langle u, v\rangle$, respectively, as follows:

$$
\begin{aligned}
& d\left([\langle u, v\rangle]^{\alpha}\right)=[\langle u, v\rangle]_{r}^{-}(\alpha)-[\langle u, v\rangle]_{l}^{-}(\alpha), \\
& d\left([\langle u, v\rangle]^{\alpha}\right)=[\langle u, v\rangle]_{r}^{+}(\alpha)-[\langle u, v\rangle]_{l}^{+}(\alpha) .
\end{aligned}
$$

Proposition 1. Let $\alpha, \beta \in[0,1]$ and $\langle u, v\rangle \in \mathbb{\mathbb { F }}^{1}$; then,

(1) $[\langle u, v\rangle]_{\alpha} \subset[\langle u, v\rangle]^{\alpha}$

(2) $[\langle u, v\rangle]_{\alpha}$ et $[\langle u, v\rangle]^{\alpha}$ are nonempty compact convex sets

(3) If $\alpha \leq \beta$, then $[\langle u, v\rangle]^{\beta} \subset[\langle u, v\rangle]^{\alpha}$ and $[\langle u, v\rangle]_{\beta}$ $\subset[\langle u, v\rangle]_{\alpha}$

(4) If $\alpha_{n} \nearrow \alpha$, then $[\langle u, v\rangle]^{\alpha}=\cap_{n}[\langle u, v\rangle]^{\alpha_{n}}$ and $[\langle u, v\rangle]_{\alpha}$ $=\cap_{n}[\langle u, v\rangle]_{\alpha_{n}}$.

Conversely, let $\alpha \in[0,1]$, and we put

$$
\begin{aligned}
& M_{\alpha}=\{x \in \mathbb{R}: u(x) \geq \alpha\}, \\
& M^{\alpha}=\{x \in \mathbb{R}: v(x) \leq 1-\alpha\} .
\end{aligned}
$$

Lemma 1 (see [17]). Let $\left\{M^{\alpha}: \alpha \in[0,1]\right\}$ and $\left\{M_{\alpha}: \alpha \in\right.$ $[0,1]\}$ be two subset of $\mathbb{R}$ verify (1)-(4) of Proposition 1 ; if $u$ and $v$ are defined by

$$
\begin{aligned}
& u(x)= \begin{cases}0 & \text { if } x \notin M_{0}, \\
\sup \left\{\alpha \in[0,1]: x \in M_{\alpha}\right\} & \text { if } x \in M_{0},\end{cases} \\
& v(x)= \begin{cases}1 & \text { if } x \notin M^{0}, \\
1-\sup \left\{\alpha \in[0,1]: x \in M^{\alpha}\right\} & \text { if } x \in M^{0},\end{cases}
\end{aligned}
$$

then $\langle u, v\rangle \in \mathbb{\mathbb { F } ^ { 1 }}$.

Lemma 2 (see [4]). Let I be a dense subset in $[0,1]$.

If $[\langle u, v\rangle]_{\alpha}=[\langle w, z\rangle]_{\alpha}$ and $[\langle u, v\rangle]^{\alpha}=[\langle w, z\rangle]^{\alpha}, \forall \alpha \in$ $I$, then $\langle u, v\rangle=\langle w, z\rangle$.

Definition 4 (see [18]). Let $\left\langle u_{1}, v_{1}\right\rangle,\left\langle u_{2}, v_{2}\right\rangle \in \mathbb{\mathbb { F } ^ { 1 }}$; if there exists $\langle w, z\rangle \in \mathbb{\mathbb { F } ^ { 1 }}$ such that

$$
\left\langle u_{1}, v_{1}\right\rangle=\left\langle u_{2}, v_{2}\right\rangle+\langle w, z\rangle
$$

then $\langle w, z\rangle$ is called Hukuhara difference of $\left\langle u_{1}, v_{1}\right\rangle$ and $\left\langle u_{2}, v_{2}\right\rangle$ denoted by $\left\langle u_{1}, v_{1}\right\rangle \ominus_{H}\left\langle u_{2}, v_{2}\right\rangle$.

Definition 5 (see $[18,19])$. The generalized Hukuhara difference of two intuitionistic fuzzy number $\left\langle u_{1}, v_{1}\right\rangle,\left\langle u_{2}, v_{2}\right\rangle \in \mathbb{\mathbb { F } ^ { 1 }}$ is as follows: 


$$
\left\langle u_{2}, v_{2}\right\rangle \ominus_{g H}\left\langle u_{1}, v_{1}\right\rangle=\left\langle u_{3}, v_{3}\right\rangle \Longleftrightarrow\left\{\begin{array}{l}
\text { i) }\left\langle u_{2}, v_{2}\right\rangle=\left\langle u_{1}, v_{1}\right\rangle+\left\langle u_{3}, v_{3}\right\rangle, \\
\text { or } \\
\text { ii) }\left\langle u_{1}, v_{1}\right\rangle=\left\langle u_{2}, v_{2}\right\rangle+(-1)\left\langle u_{3}, v_{3}\right\rangle .
\end{array}\right.
$$

Definition 6 (see [17]). Let $f:[a, b] \longrightarrow \mathbb{\mathbb { F } ^ { 1 }}$ and $t_{0} \in[a, b]$. We say that $f$ is generalized Hukuhara differentiable at $t_{0}$ if there exists $f^{\prime}\left(t_{0}\right) \in \mathbb{\mathbb { F } ^ { 1 }}$ such that

$$
f^{\prime}\left(t_{0}\right)=\lim _{h \longrightarrow 0^{+}} \frac{f\left(t_{0}+h\right) \ominus_{g H} f\left(t_{0}\right)}{h}=\lim _{h \longrightarrow 0^{-}} \frac{f\left(t_{0}\right) \ominus_{g H} f\left(t_{0}-h\right)}{h} .
$$

Definition 7 (see [17]). A function $F:[a, b] \longrightarrow \mathbb{Q} \mathbb{F}^{1}$ is strongly measurable if the set-valued mappings
$F_{\alpha}:[a, b] \longrightarrow \mathscr{P}_{K}(\mathbb{R})$ defined by $F_{\alpha}(t)=[F(t)]^{\alpha}$ and $F^{\alpha}:[a, b] \longrightarrow \mathscr{P}_{K}(\mathbb{R})$ defined by $F^{\alpha}(t)=[F(t)]_{\alpha}$ are Lebesgue measurable, for all $\alpha \in[0,1]$, where $\mathscr{P}_{K}(\mathbb{R})$ is the set of all closed and bounded intervals of $\mathbb{R}$.

Definition 8 (see $[17,19])$. Let $F:[a, b] \longrightarrow \mathbb{F ^ { 1 }}$. We say that $F$ is integrable on $[a, b]$ if there exists $\langle u, v\rangle \in \mathbb{\mathbb { F } ^ { 1 }}$ such that, for each $\alpha \in[0,1]$,

$$
\begin{aligned}
{\left[\int_{a}^{b} F(t) \mathrm{d} t\right]^{\alpha} } & =\left\{\int_{a}^{b} f(t) \mathrm{d} t \mid f:[a, b] \longrightarrow \mathbb{R} \text { is a measurable selection for } F_{\alpha}\right\} \\
{\left[\int_{a}^{b} F(t) \mathrm{d} t\right]_{\alpha} } & =\left\{\int_{a}^{b} f(t) \mathrm{d} t \mid f:[a, b] \longrightarrow \mathbb{R} \text { is a measurable selection for } F_{\alpha}\right\}, \\
{[\langle u, v\rangle]^{\alpha} } & =\left[\int_{a}^{b} F(t) \mathrm{d} t\right]^{\alpha}, \\
{[\langle u, v\rangle]_{\alpha} } & =\left[\int_{a}^{b} F(t) \mathrm{d} t\right]_{\alpha},
\end{aligned}
$$

and we write $\int_{a}^{b} F(t) \mathrm{d} t=\langle u, v\rangle$.

Let $d_{p}: \mathbb{a} \mathbb{F}^{1} \times \mathbb{Q} \mathbb{F}^{1} \longrightarrow[0,+\infty]$ be a mapping defined by

$$
\begin{aligned}
d_{p}(\langle u, v\rangle,\langle w, z\rangle)= & \frac{1}{4} \int_{0}^{1}\left|[\langle u, v\rangle]_{r}^{+}(\alpha)-[\langle w, z\rangle]_{r}^{+}(\alpha)\right|^{p} \mathrm{~d} \alpha \\
& +\frac{1}{4} \int_{0}^{1}\left|[\langle u, v\rangle]_{l}^{+}(\alpha)-[\langle w, z\rangle]_{l}^{+}(\alpha)\right|^{p} \mathrm{~d} \alpha \\
& +\frac{1}{4} \int_{0}^{1}\left|[\langle u, v\rangle]_{r}^{-}(\alpha)-[\langle w, z\rangle]_{r}^{-}(\alpha)\right|^{p} \mathrm{~d} \alpha \\
& \left.+\frac{1}{4} \int_{0}^{1}\left|[\langle u, v\rangle]_{l}^{-}(\alpha)-[\langle w, z\rangle]_{l}^{-}(\alpha)\right|^{p} \mathrm{~d} \alpha\right)^{1 / p} \quad \text { for } p \in[1,+\infty[. \\
\text { and } d_{\infty}(\langle u, v\rangle,\langle w, z\rangle)= & \frac{1}{4} \sup _{0 \leq \alpha \leq 1}\left|[\langle u, v\rangle]_{r}^{+}(\alpha)-[\langle w, z\rangle]_{r}^{+}(\alpha)\right| \\
& +\frac{1}{4} \sup _{0 \leq \alpha \leq 1}\left|[\langle u, v\rangle]_{l}^{+}(\alpha)-[\langle w, z\rangle]_{l}^{+}(\alpha)\right| \\
& +\frac{1}{4} \sup _{0 \leq \alpha \leq 1}\left|[\langle u, v\rangle]_{r}^{-}(\alpha)-[\langle w, z\rangle]_{r}^{-}(\alpha)\right| \\
& +\frac{1}{4} \sup _{0 \leq \alpha \leq 1}\left|[\langle u, v\rangle]_{l}^{-}(\alpha)-[\langle w, z\rangle]_{l}^{-}(\alpha)\right|
\end{aligned}
$$


Then, we have the following result.

Proposition 2 (see [20]). The space $\left(\square \mathbb{F}^{1}, d_{p}\right)$ is an intuitionistic fuzzy complete metric space $\forall p \in[1,+\infty]$.

In the following sections, we will need some notations and definitions.

(i) We denote by $C\left([0, T], \mathbb{F}^{1}\right)$ the space of all intuitionistic fuzzy continuous functions from $[0, T]$ to $\mathbb{\mathbb { F } ^ { 1 }}$

(ii) We denote by $L\left([0, T], \mathbb{\mathbb { F } ^ { 1 }}\right)$ the space of all intuitionistic fuzzy integrable functions on $[0, T]$

Remark 2. Let $x, y \in C\left([0, T], \square \mathbb{F}^{-1}\right)$.

It is easy to see that the space $\left(C\left([0, T], \mathbb{a} \mathbb{F}^{1}\right), D_{p}\right)$ is a Banach space, where

$$
D_{p}(x, y)=\sup _{s \in[0, T]} d_{p}(x(s), y(s)) .
$$

2.1. Fractional Integral and Fractional Derivatives of an Intuitionistic Fuzzy Function

Definition 9 (see [20]). Let $F(t) \in L\left([0, T], \mathbb{\mathbb { F } ^ { 1 }}\right)$.

The intuitionistic fuzzy fractional integral of order $q \in[0,1]$ of $F$, denoted by

$$
I^{q} F(t)=\frac{1}{\Gamma(q)} \int_{0}^{t}(t-s)^{q-1} F(s) \mathrm{d} s
$$

is defined by

$$
\begin{aligned}
& {\left[I^{q} F(t)\right]^{\alpha}=\left[I^{\alpha} F_{l}^{-}(t ; \alpha), I^{q} F_{r}^{-}(t ; \alpha)\right],} \\
& {\left[I^{q} F(t)\right]_{\alpha}=\left[I^{\alpha} F_{l}^{+}(t ; \alpha), I^{q} F_{r}^{+}(t ; \alpha)\right],}
\end{aligned}
$$

where $\Gamma($.$) is the Euler gamma function.$
Proposition 3 (see [20]). Let $F, G \in L\left([0, T], \square \mathbb{F}^{1}\right), q \in[0,1]$ and $a \in \mathbb{\mathbb { F } ^ { 1 }}$; then, we have

(1) $I^{q}(a F)(t)=a I^{q} F(t)$

(2) $I^{q}(F+G)(t)=I^{q} F(t)+I^{q} G(t)$

(3) $I^{q_{1}} I^{q_{2}} F(t)=I^{q_{1}+q_{2}} F(t)$, where $\left(q_{1}, q_{2}\right) \in[0,1]^{2}$

Definition 10 (see $[20])$. Let $F \in C\left([0, T], \mathbb{Q} \mathbb{F}^{1}\right) \cap L([0, T]$, $\left.\square \mathbb{F}^{1}\right)$.

The function $F$ is called intuitionistic fuzzy Caputo fractional differentiable of order $0<q<1$ at $x$ if there exists an element ${ }^{c} D^{q} F(x) \in \mathbb{\mathbb { F } ^ { 1 }}$ such that

$$
{ }^{c} D^{q} F(t)=\frac{1}{\Gamma(q)} \int_{0}^{t}(t-s)^{q-1} F^{\prime}(s) \mathrm{d} s .
$$

Example 1. Consider the intuitionistic fuzzy function $\langle u, v\rangle(t)=t C$, where $C=\left(a_{1} ; a_{2} ; a_{3} ; a_{4} ; a_{1}^{\prime} ; a_{2} ; a_{3} ; a_{4}^{\prime}\right)$ is a trapezoidal intuitionistic fuzzy number and $0<q<1$.

In this example, we calculate the intuitionistic fuzzy Caputo fractional derivative of the function $\langle u, v\rangle(t)$. For this purpose, we start by giving the gH-derivative of $\langle u, v\rangle(t)$ as follows:

$$
\begin{aligned}
\langle u, v\rangle^{\prime}(t) & =\lim _{h \longrightarrow 0} \frac{\langle u, v\rangle(t+h) \ominus_{g H}\langle u, v\rangle(t)}{h} \\
& =\lim _{h \rightarrow 0} \frac{(t+h) C \ominus_{g H} t C}{h}=C .
\end{aligned}
$$

This implies that

$$
\langle u, v\rangle^{\prime}(t)=C .
$$

Since $[C]^{\alpha}=\left[a_{2}-\alpha\left(a_{2}-a_{1}^{\prime}\right), a_{3}+\alpha\left(a_{4}-a_{3}\right)\right] \quad$ and $[C]_{\alpha}=\left[a_{1}+\alpha\left(a_{2}-a_{1}\right), a_{4}-\alpha\left(a_{4}^{\prime}-a_{3}\right)\right]$, then we have

$$
\begin{aligned}
{\left[{ }^{c} D^{q}\langle u, v\rangle(t)\right]^{\alpha} } & =\left[I^{1-q}\left(\langle u, v\rangle^{\prime}(t)\right)\right]^{\alpha}=\left[I^{1-q} C\right]^{\alpha}, \\
{\left[{ }^{c} D^{q}\langle u, v\rangle(t)\right]_{\alpha} } & =\left[I^{1-q}\left(\langle u, v\rangle^{\prime}(t)\right)\right]^{\alpha}=\left[I^{1-q} C\right]_{\alpha}, \\
{\left[I^{1-q} C\right]^{\alpha} } & =\frac{1}{\Gamma(q)} \int_{0}^{t}(t-s)^{q^{-1}}\left[a_{2}-\alpha\left(a_{2}-a_{1}^{\prime}\right), a_{3}+\alpha\left(a_{4}-a_{3}\right)\right] \mathrm{d} s, \\
{\left[I^{1-q} C\right]_{\alpha} } & =\frac{1}{\Gamma(q)} \int_{0}^{t}(t-s)^{q-1}\left[a_{1}+\alpha\left(a_{2}-a_{1}\right), a_{4}-\alpha\left(a_{4}^{\prime}-a_{3}\right)\right] \mathrm{d} s, \\
{\left[I^{1-q} C\right]^{\alpha} } & =\frac{t^{q}}{\Gamma(q) \times q}\left[a_{2}-\alpha\left(a_{2}-a_{1}^{\prime}\right), a_{3}+\alpha\left(a_{4}-a_{3}\right)\right], \\
{\left[I^{1-q} C\right]_{\alpha} } & =\frac{t^{q}}{\Gamma(q) \times q}\left[a_{1}+\alpha\left(a_{2}-a_{1}\right), a_{4}-\alpha\left(a_{4}^{\prime}-a_{3}\right)\right], \\
{\left[I^{1-q} C\right]^{\alpha} } & =\frac{t^{q}}{\Gamma(q+1)}[C]^{\alpha}, \\
{\left[I^{1-q} C\right]_{\alpha} } & =\frac{t^{q}}{\Gamma(q+1)}[C]_{\alpha} .
\end{aligned}
$$


Thus,

$$
\begin{aligned}
& { }^{c} D^{q}\langle u, v\rangle(t)=\frac{t^{q}}{\Gamma(q+1)} C, \\
& { }^{c} D^{q}\langle u, v\rangle(t)=\frac{t^{q-1}}{\Gamma(q+1)}\langle u, v\rangle(t) .
\end{aligned}
$$

Theorem 1 (Schaefer's fixed-point theorem (see [21])). Let $\mathbf{P}$ be a continuous and compact mapping of a Banach space $X$ into itself such that the set

$$
\{x \in X: x=\lambda \mathbf{P} x \quad \text { forsome } 0 \leq \lambda \leq 1\}
$$

is bounded; then, mapping $\boldsymbol{P}$ has a fixed point.

\section{Intuitionistic Fuzzy Local Fractional Boundary Value Problems}

Definition 11. A function $X:[0, T] \longrightarrow \mathbb{F}^{-1}$ is said to be a solution of problem (1) if $X \in C^{1}\left([0, T], \mathbb{F}^{1}\right)$ such that $X(0)=a X(T)$ and $D^{\alpha} X(t)=F(t, X(t))$.

Definition 12 (see [6]). An intuitionistic fuzzy function $X:[0, T] \longrightarrow \mathbb{Q} \mathbb{F}^{1}$ is called d-increasing (d-decreasing) on $[0, T]$ if, for every $r \in[0,1]$, the real function $t \longrightarrow d\left([X(t)]^{r} \cup[X(t)]_{r}\right.$ ) is nondecreasing (nonincreasing), respectively.

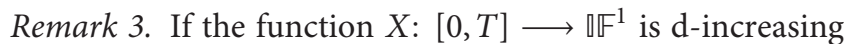
or d-decreasing on $[0, T]$, then we say that $X(t)$ is d-monotone on $[0, T]$.

For the existence of solutions for problem (1), we need the following lemma.

Lemma 3 (see [6]). Let $\alpha \in] 0,1\left[\right.$ and $H:[0, T] \longrightarrow \mathbb{F}^{-1}$ be continuous such that $t \longrightarrow I^{\alpha} H(t)$ is d-increasing function on $[0, T]$.

A d-monotone intuitionistic fuzzy function $X(t) \in C\left([0, T], \square \mathbb{F}^{1}\right)$ is a solution of the fractional integral equation,

$$
X(t) \ominus_{g H} X_{0}=\frac{1}{\Gamma(\alpha)} \int_{0}^{t}(t-s)^{\alpha-1} H(s) \mathrm{d} s,
$$

if and only if it is a solution of the following initial value problem:

$$
\left\{\begin{array}{l}
{ }^{c} D^{\alpha} X(t)=H(t) ; \quad t \in[0, T] \\
X(0)=X_{0}
\end{array}\right.
$$

As a consequence of Lemma 3, we have the following result.

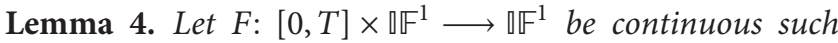
that $t \longrightarrow I^{\alpha} F(t, X(t))$ is d-increasing function on $[0, T]$.

A $d$-monotone intuitionistic fuzzy function $X(t)$ is a solution of the fractional integral equation,

$$
X(t) \ominus_{g H} \frac{a}{(1-a) \Gamma(\alpha)} \int_{0}^{T}(T-s)^{\alpha-1} F(s, X(s)) \mathrm{d} s=\frac{1}{\Gamma(\alpha)} \int_{0}^{t}(t-s)^{\alpha-1} F(s, X(s)) \mathrm{d} s,
$$

if and only if is a solution of the fractional boundary value problem (1).
Theorem 2. Assume that there exists a positive constant $k$ such that

$$
d_{p}\left(F(t, X), F(t, Y)<k d_{p}(X, Y), \quad \text { for each } X, Y \in C\left([0, T], \square \mathbb{F}^{1}\right) \text { and } t \in[0, T]\right. \text {. }
$$

In addition, if

$$
\frac{k T^{\alpha}(1+|a| /|1-a|)}{\Gamma(\alpha+1)}<1,
$$

then problem (1) has a unique solution.
Proof. For this purpose, we transform problem (1) into a fixed-point problem defined on $[0, T]$.

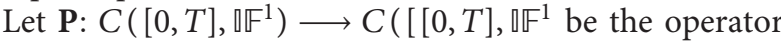
defined as follows:

$$
\mathbf{P} X(t) \ominus_{g H} \frac{a}{(1-a) \Gamma(\alpha)} \int_{0}^{T}(T-s)^{\alpha-1} F(s, X(s)) \mathrm{d} s=\frac{1}{\Gamma(\alpha)} \int_{0}^{t}(t-s)^{\alpha-1} F(s, X(s)) \mathrm{d} s .
$$

Let $X, Y \in C\left([0, T], \square \mathbb{F}^{-1}\right)$; then, we have 


$$
\begin{aligned}
& d_{p}(\mathbf{P} X(t), \mathbf{P} Y(t)) \leq \frac{1}{\Gamma(\alpha)} \int_{0}^{t}(t-s)^{\alpha-1} d_{p}(F(t, X(t)), F(t, Y(t))) \mathrm{d} s \\
& +\frac{|a|}{|1-a| \Gamma(\alpha)} \int_{0}^{T}(T-s)^{\alpha-1} d_{p}(F(s, X(s)), F(s, Y(s))) \mathrm{d} s, \\
& d_{p}(\mathbf{P} X(t), \mathbf{P Y}(t)) \leq \frac{k}{\Gamma(\alpha)} \int_{0}^{t}(t-s)^{\alpha-1} d_{p}(X(t), Y(t)) \mathrm{d} s \\
& +\frac{k|a|}{|1-a| \Gamma(\alpha)} \int_{0}^{T}(T-s)^{\alpha-1} d_{p}(X(s), Y(s)) \mathrm{d} s, \\
& d_{p}(\mathbf{P} X(t), \mathbf{P} Y(t)) \leq \frac{k}{\Gamma(\alpha)} \sup _{s \in[0, T]} d_{p}(X(s), Y(s)) \int_{0}^{t}(t-s)^{\alpha-1} \mathrm{~d} s \\
& +\frac{k|a|}{|1-a| \Gamma(\alpha)} \sup _{s \in[0, T]} d_{p}(X(s), Y(s)) \int_{0}^{T}(T-s)^{\alpha-1} \mathrm{~d} s \\
& d_{p}(\mathrm{PX}(t), \mathrm{PY}(t)) \leq \frac{k T^{\alpha}(1+|a| /|1-a|)}{\alpha \Gamma(\alpha)} \sup _{s \in[0, T]} d_{p}(X(s), Y(s)), \\
& d_{p}(\mathbf{P} X(t), \mathbf{P} Y(t)) \leq \frac{k T^{\alpha}(1+|a| /|1-a|)}{\Gamma(\alpha+1)} \sup _{s \in[0, T]} d_{p}(X(s), Y(s)) .
\end{aligned}
$$

Thus,

$$
\begin{gathered}
\sup _{s \in[0, T]} d_{p}(\mathbf{P} X(t), \mathbf{P} Y(t)) \leq \frac{k T^{\alpha}(1+|a| /|1-a|)}{\Gamma(\alpha+1)} \sup _{s \in[0, T]} d_{p}(X(t), Y(t)) \\
D_{p}(\mathbf{P} X, \mathbf{P Y}) \leq \frac{k T^{\alpha}(1+|a| /|1-a|)}{\Gamma(\alpha+1)} D_{p}(X, Y) .
\end{gathered}
$$

As a consequence of Banach fixed-point theorem, we can deduce that the operator $\mathbf{P}$ has a unique fixed point $X$ which is the solution of problem (1).

Theorem 3. Assume that

$\left(H_{1}\right)$ The intuitionistic fuzzy function $F:[0, T] \times \mathbb{Q} \mathbb{F}^{1} \longrightarrow \mathbb{\mathbb { F } ^ { 1 }}$ is continuous.

$\left(\mathrm{H}_{2}\right)$ There exists a positive constant $M$ such that

$$
d_{p}\left(F(t, X), 0_{\mathbb{I F}}\right)<M \quad \forall(t, X) \in[0, T] \times C\left([0, T], \mathbb{\mathbb { F } ^ { 1 }}\right) .
$$

Then, problem (1) has at least one solution.

Proof. To show that problem (1) has at least one solution defined on $[0, T]$, we use Schaefer's fixed-point theorem [22]. For this purpose, to prove that the operator $\mathbf{P}$ defined 
above has a fixed point, the proof of this theorem will be given in several steps.

Step 1: let us show that $\mathbf{P}$ is continuous.
Let $\left(X_{n}\right)_{n} \subset C\left([0, T], \square \mathbb{F}^{1}\right)$ such that $X_{n}$ converges to $X$ in $C\left(\left[[0, T], \square \mathbb{F}^{1}\right)\right.$ and $t \in[0, T]$; we have

$$
\begin{aligned}
d_{p}\left(\mathbf{P} X_{n}(t), \mathbf{P} X(t)\right) \leq & \frac{1}{\Gamma(\alpha)} \int_{0}^{t}(t-s)^{\alpha-1} d_{p}\left(F\left(t, X_{n}(t)\right), F(t, X(t))\right) \mathrm{d} s \\
& +\frac{|a|}{|1-a| \Gamma(\alpha)} \int_{0}^{T}(T-s)^{\alpha-1} d_{p}\left(F\left(s, X_{n}(s)\right), F(s, X(s))\right) \mathrm{d} s, \\
d_{p}\left(\mathbf{P} X_{n}(t), \mathbf{P} X(t)\right) \leq & \frac{1}{\Gamma(\alpha)} \int_{0}^{t}(t-s)^{\alpha-1} \sup _{s \in[0, T]} d_{p}\left(F\left(s, X_{n}(s)\right), F(s, X(s))\right) \mathrm{d} s \\
& \left.+\frac{|a|}{|1-a| \Gamma(\alpha)} \int_{0}^{T}(T-s)^{\alpha-1} \sup _{s \in[0, T]} d_{p}\left(F(s), X_{n}(s)\right), F(s, X(s))\right) \mathrm{d} s, \\
d_{p}\left(\mathbf{P} X_{n}(t), \mathbf{P} X(t)\right) \leq & \frac{T^{\alpha}(1+|a| /|1-a|)}{\alpha \Gamma(\alpha)} \sup _{s \in[0, T]} d_{p}\left(F\left(s, X_{n}(s)\right), F(s, X(s))\right), \\
d_{p}\left(\mathbf{P} X_{n}(t), \mathbf{P} X(t)\right) \leq & \frac{T^{\alpha}(1+|a| /|1-a|)}{\Gamma(\alpha+1)} \sup _{s \in[0, T]} d_{p}\left(F\left(s, X_{n}(s)\right), F(s, X(s))\right) .
\end{aligned}
$$

Thus,

$$
\sup _{s \in[0, T]} d_{p}\left(\mathbf{P} X_{n}(t), \mathbf{P} X(t)\right) \leq \frac{T^{\alpha}(1+|a| /|1-a|)}{\Gamma(\alpha+1)} \sup _{s \in[0, T]} d_{p}\left(F\left(t, X_{n}(t)\right), F(t, X(t))\right) .
$$

Since $F$ is continuous, we obtain

$$
\lim _{n \rightarrow \infty} \sup _{t \in[0, T]} d_{p}\left(\mathbf{P} X_{n}(t), \mathbf{P} X(t)\right) \leq \frac{T^{\alpha}(1+|a| /|1-a|)}{\Gamma(\alpha+1)} \lim _{n \rightarrow \infty} \sup _{t \in[0, T]} d_{p}\left(F\left(t, X_{n}(t)\right), F(t, X(t))\right)=0
$$

which implies that

$$
\lim _{n \rightarrow \infty} \sup _{t \in[0, T]} d_{p}\left(\mathbf{P} X_{n}(t), \mathbf{P} X(t)\right)=0 .
$$

$$
X \in B_{\rho}=\left\{X \in C\left([0, T], \mathbb{\mathbb { F } ^ { 1 }}\right): \sup _{t \in[0, T]} d_{p}\left(X(t), 0_{\mathbb{\mathbb { F }}}\right) \leq \rho\right\}
$$

Finally, the operator $\mathbf{P}$ is continuous on $C\left([0, T], \square \mathbb{F}^{1}\right)$.

Step 2: let us also show that $\mathbf{P} B_{\rho}$ is bounded and equicontinuous on $C\left([0, T], \square \mathbb{F}^{1}\right)$.

It is enough to prove that, for any $\rho>0$, there exists a we have

$$
\sup _{t \in[0, T]} d_{p}\left(\mathbf{P} X(t), 0_{\mathbb{E}}\right)<\delta .
$$

Let $t \in[0, T]$, and we have positive real constant $\delta>0$ such that, for each

$$
\begin{aligned}
d_{p}\left(\mathbf{P} X(t), 0_{\mathbb{V}}\right) \leq & \frac{1}{\Gamma(\alpha)} \int_{0}^{t}(t-s)^{\alpha-1} d_{p}\left(F(t, X(t)), 0_{\mathbb{I F}}\right) \mathrm{d} s \\
& +\frac{|a|}{|1-a| \Gamma(\alpha)} \int_{0}^{T}(T-s)^{\alpha-1} d_{p}\left(F(s, X(s)), 0_{\mathbb{E}}\right) \mathrm{d} s .
\end{aligned}
$$


By using $H_{2}$, we have

$$
\begin{gathered}
d_{p}\left(\mathbf{P} X(t) 0_{\mathbb{V}}\right) \leq \frac{M}{\Gamma(\alpha)} \int_{0}^{t}(t-s)^{\alpha-1} \mathrm{~d} s+\frac{M|a|}{|1-a| \Gamma(\alpha)} \int_{0}^{T}(T-s)^{\alpha-1} \mathrm{~d} s \\
d_{p}\left(\mathbf{P} X(t) 0_{\mathbb{V} F}\right) \leq \frac{\mathrm{MT}^{\alpha}}{\alpha \Gamma(\alpha)}+\frac{\mathrm{MT}^{\alpha}|a|}{|1-a| \alpha \Gamma(\alpha)}, \\
\sup _{t \in[0, T]} d_{p}\left(\mathbf{P} X(t) 0_{\mathbb{D} F}\right) \leq \frac{\mathrm{MT}^{\alpha}}{\alpha \Gamma(\alpha)}+\frac{\mathrm{MT}^{\alpha}|a|}{|1-a| \alpha \Gamma(\alpha)}:=\delta .
\end{gathered}
$$

Thus, we have that $\mathbf{P} B_{\rho} \subset B_{\delta}$, which implies that $\mathbf{P} B_{\rho}$ is bounded.

Let $X \in B_{\rho}$ and $t_{1}, t_{2} \in[0, T]$ such that $t_{1}<t_{2}$; then, we

Let $t \in[0, T]$.

$$
d_{p}\left(\mathbf{P} X\left(t_{1}\right), \mathbf{P} X\left(t_{2}\right)\right) \leq \frac{1}{\Gamma(\alpha)} d_{p}\left(\int_{0}^{t_{1}}\left(t_{1}-s\right)^{\alpha-1} F(s, X(s)) \mathrm{d} s, \int_{0}^{t_{2}}\left(t_{2}-s\right)^{\alpha-1} F(s, X(s)) \mathrm{d} s\right) .
$$

Since

$$
\int_{0}^{t_{2}}\left(t_{2}-s\right)^{\alpha-1} F(s, X(s)) \mathrm{d} s=\int_{0}^{t_{1}}\left(t_{2}-s\right)^{\alpha-1} F(s, X(s)) \mathrm{d} s+\int_{t_{1}}^{t_{2}}\left(t_{2}-s\right)^{\alpha-1} F(s, X(s)) \mathrm{d} s
$$

then

$$
\begin{aligned}
d_{p}\left(\mathbf{P} X\left(t_{1}\right), \mathbf{P} X\left(t_{2}\right)\right) \leq & \frac{1}{\Gamma(\alpha)} \int_{t_{0}}^{t_{1}}\left|\left(t_{1}-s\right)^{\alpha-1}-\left(t_{1}-s\right)^{\alpha-1}\right| d_{p}\left(F(s, X(s)), 0_{\mathbb{\mathbb { F } ^ { 1 }}}\right) \\
& +\int_{t_{1}}^{t_{2}}\left(t_{2}-s\right)^{\alpha-1} d_{p}\left(F(s, X(s)) \mathrm{d} s, 0_{\mathbb{a} \mathbb{F}^{1}}\right) \mathrm{d} s .
\end{aligned}
$$

By using $H_{2}$, it follows that

$$
\begin{gathered}
d_{p}\left(\left(\mathbf{P} X\left(t_{1}\right), \mathbf{P} X\left(t_{2}\right)\right) \leq \frac{M}{\Gamma(\alpha)} \int_{t_{0}}^{t_{1}}\left|\left(t_{1}-s\right)^{\alpha-1}-\left(t_{1}-s\right)^{\alpha-1}\right| \mathrm{d} s+\frac{M}{\Gamma(\alpha)} \int_{t_{1}}^{t_{1} t_{2}}\left(t_{2}-s\right)^{\alpha-1} \mathrm{~d} s,\right. \\
d_{p}\left(\mathbf{P} X\left(t_{1}\right), \mathbf{P} X\left(t_{2}\right)\right) \leq \frac{M}{\alpha \Gamma(\alpha)}\left(\left(t_{2}-t_{1}\right)^{\alpha}+t_{1}^{\alpha}-t_{2}^{\alpha}\right)+\frac{M}{\alpha \Gamma(\alpha)}\left(t_{2}-t_{1}\right)^{\alpha} . \\
\lim _{t_{1} \longrightarrow t_{2}} d_{p}\left(\mathbf{T} X\left(t_{1}\right), \mathbf{P} X\left(t_{2}\right)\right)=0,
\end{gathered}
$$

Thus,

$d_{p}\left(\mathbf{P} X\left(t_{1}\right), \mathbf{P} X\left(t_{2}\right)\right) \leq \frac{M}{\Gamma(\alpha+1)}\left(\left(t_{2}-t_{1}\right)^{\alpha}+\left(t_{2}^{\alpha}-t_{1}^{\alpha}\right)\right)$.

Hence, which shows that $\mathbf{P} B$ is equicontinuous; by using Arzela-Ascoli theorem [23], we deduce that $\mathbf{P} B_{\rho}$ is relatively compact, and we can conclude from Step 1 and Step 2 that the operator $\mathbf{P}$ is continuous and completely continuous. 
Step 3 : let us we show that the set $E_{\lambda}$, defined as follows,

$$
E_{\lambda}=\left\{X \in C\left([0, T], \mathbb{} \mathbb{F}^{1}\right): X=\lambda \mathbf{P} X \quad \text { for some } 0 \leq \lambda \leq 1\right\},
$$

is bounded.

Let $X \in E_{\lambda}$; then, $X=\lambda \mathbf{P} X$, for some $\lambda \in[0,1]$. For each $t \in[0, T]$, we have

$$
X(t) \ominus_{g H} \frac{\lambda a}{(1-a) \Gamma(\alpha)} \int_{0}^{T}(T-s)^{\alpha-1} F(s, X(s)) \mathrm{d} s=\frac{\lambda}{\Gamma(\alpha)} \int_{0}^{t}(t-s)^{\alpha-1} F(s, X(s)) \mathrm{d} s .
$$

Let $t \in[0, T]$, and by using $H_{3}$, we have

$$
\begin{aligned}
& d_{p}\left(\mathbf{P} X(t), 0_{\mathbb{a}}\right) \leq \frac{1}{\Gamma(\alpha)} \int_{0}^{t}(t-s)^{\alpha-1} d_{p}\left(F(t, X(t)), 0_{\mathbb{a F}}\right) \mathrm{d} s+\frac{|a|}{|1-a| \Gamma(\alpha)} \int_{0}^{T}(T-s)^{\alpha-1} d_{p}\left(F(s, X(s)), 0_{\mathbb{a F}}\right) \mathrm{d} s \\
& d_{p}\left(\mathbf{P} X(t), 0_{\mathbb{E}}\right) \leq \frac{M}{\Gamma(\alpha)} \int_{0}^{t}(t-s)^{\alpha-1} \mathrm{~d} s+\frac{M|a|}{|1-a| \Gamma(\alpha)} \int_{0}^{T}(T-s)^{\alpha-1} \mathrm{~d} s, \\
& d_{p}\left(\mathbf{P} X(t), 0_{\mathbb{V E}}\right) \leq \frac{\mathrm{MT}^{\alpha}}{\alpha \Gamma(\alpha)}+\frac{\mathrm{MT}^{\alpha}|a|}{|1-a| \alpha \Gamma(\alpha)}, \\
& \sup _{t \in[0, T]} d_{p}\left(\mathbf{P} X(t), 0_{\mathbb{V}}\right) \leq \frac{\mathrm{MT}^{\alpha}}{\Gamma(\alpha+1)}+\frac{|a| \mathrm{MT}^{\alpha}}{|1-a| \Gamma(\alpha+1)}<\infty,
\end{aligned}
$$

which shows that the set $E_{\lambda}$ is bounded.

Finally, as a consequence of Schaefer's fixed-point theorem, we deduce that $\mathbf{P}$ has a fixed point which is a solution of problem (1).

\section{Intuitionistic Fuzzy Nonlocal Fractional Boundary Value Problems}

In this section, we present some existence and uniqueness results for nonlocal intuitionistic fuzzy fractional problem (2). The nonlocal conditions were initiated by Byszewski [24]

$$
d_{p}\left(F(t, X), F(t, Y)<k d_{p}(X, Y)\right.
$$

(3) There exists a positive constant $L$ such that

$$
d_{p}\left(G(U), G(V) \leq L d_{p}(U, V) \quad \text { for each } U, V \in C\left([0, T], \mathbb{\mathbb { F } ^ { 1 }}\right) .\right.
$$

In addition, if

$$
L+\frac{k T^{\alpha}}{\Gamma(\alpha+1)}<1
$$

when he proved the existence and uniqueness of mild and classical solutions of nonlocal Cauchy problems. Some physical phenomena are described the nonlocal condition; see $[25,26]$, for more details.

Theorem 5. Assume that

(1) The intuitionistic fuzzy function $F:[0, T] \times \mathbb{\mathbb { F } ^ { 1 }} \longrightarrow \mathbb{\mathbb { F } ^ { 1 }}$ is continuous.

(2) There exists a positive constant $k$ such that

$X, Y \in C\left([0, T], \square \mathbb{F}^{1}\right)$ and $t \in[0, T]$.

then problem (2) has a unique solution.

Proof. For this purpose, we transform problem (2) into a fixed-point problem defined on $[0, T]$.

Let B: $C\left([0, T], \square \mathbb{F}^{1}\right) \longrightarrow C\left([0, T], \square \mathbb{F}^{1}\right.$ be the operator defined as follows:

$$
\mathbf{B} X(t) \ominus_{g H} G(X)=\frac{1}{\Gamma(\alpha)} \int_{0}^{t}(t-s)^{\alpha-1} F(s, X(s)) \mathrm{d} s .
$$


Let $X, Y \in C\left([0, T], \square \mathbb{F}^{1}\right)$; then, we have

$$
\begin{gathered}
d_{p}(\mathbf{B} X(t), \mathbf{B} Y(t)) \leq d_{p}(G(X(t)), G(Y(t)))+\frac{1}{\Gamma(\alpha)} \int_{0}^{t}(t-s)^{\alpha-1} d_{p}(F(t, X(t)), F(t, Y(t))) \mathrm{d} s, \\
d_{p}(\mathbf{B} X(t), \mathbf{B} Y(t)) \leq L d_{p}(X(t), Y(t))+\frac{1}{\Gamma(\alpha)} \int_{0}^{t}(t-s)^{\alpha-1} d_{p}(X(t), Y(t)) \mathrm{d} s \\
d_{p}(\mathbf{B} X(t), \mathbf{B} Y(t)) \leq L d_{p}(X(t), Y(t))+\frac{1}{\Gamma(\alpha)} \int_{0}^{t}(t-s)^{\alpha-1} \mathrm{~d} s \sup _{s \in[0, T]} d_{p}(X(s), Y(s)), \\
\sup _{t \in[0, T]} d_{p}(\mathbf{B} X(t), \mathbf{B} Y(t)) \leq L \sup _{t \in[0, T]} d_{p}(X(t), Y(t))+\frac{k T^{\alpha}}{\alpha \Gamma(\alpha)} \mathrm{d} s \sup _{t \in[0, T]} d_{p}(X(t), Y(t)) \\
\sup _{t \in[0, T]} d_{p}(\mathbf{B} X(t), \mathbf{B} Y(t)) \leq\left(L+\frac{k T^{\alpha}}{\Gamma(\alpha+1)}\right) \sup _{t \in[0, T]} d_{p}(X(t), Y(t)) .
\end{gathered}
$$

Thus,

$$
D_{p}(\mathbf{B} X, \mathbf{B} Y) \leq\left(L+\frac{k T^{\alpha}}{\Gamma(\alpha+1)}\right) D_{p}(X, Y) .
$$

By using Banach fixed-point theorem, we can deduce that the operator $\mathbf{B}$ has a unique fixed point $X$ which is the solution of problem (2).

Theorem 6. Assume that

(1) The intuitionistic fuzzy function $F:[0, T] \times \mathbb{\mathbb { F } ^ { 1 }} \longrightarrow \mathbb{\mathbb { F } ^ { 1 }}$ is continuous.

(2) There exists a positive constant $M_{1}$ such that $d_{p}\left(F(t, X), 0_{\mathbb{a}}\right) \leq M_{1} \quad \forall(t, x) \in[0, T] \times \mathbb{\mathbb { F } ^ { 1 }}$.

(3) There exists a positive constant $M_{2}$ such that

$$
d_{p}\left(G(X), 0_{\mathbb{Q F}}\right) \leq M_{2} \quad \forall x \in C\left([0, T], \mathbb{Q} \mathbb{F}^{1}\right)
$$

Then, problem (2) has at least one solution.

Proof. The proof of this theorem is similar to that of Theorem 3.

\section{Illustrative Example}

In this section, we give an example to illustrate our main results.

Consider the following fractional boundary value problem:

$$
\left\{\begin{array}{l}
{ }^{c} D^{2 / 3} X(t)=\frac{e^{-t}}{9+e^{t}} X(t), \quad t \in[0,1], \\
X(0)=(-1) X(1),
\end{array}\right.
$$

where $t \longmapsto e^{t}$ is the exponential function.

Let $F(t, X(t))=e^{-t} / 9+e^{t} X(t)$ and $t \in[0,1]$; then, we have

$$
\begin{aligned}
& d_{p}(F(t, X(t)), F(t, Y(t)))=d_{p}\left(\frac{e^{-t}}{9+e^{t}} X(t), \frac{e^{-t}}{9+e^{t}} Y(t)\right), \\
& d_{p}(F(t, X(t)), F(t, Y(t))) \leq \frac{e^{-t}}{9+e^{t}} d_{p}(X(t), Y(t)),
\end{aligned}
$$

$$
d(F(t, X(t)), F(t, Y(t))) \leq \frac{1}{10} d_{p}(X(t), Y(t)) .
$$

Hence, the first condition in Theorem 2 holds with $k=1 / 10$.

It remains to check condition (29) with $T=1, a=-1$, and $\alpha=2 / 3$; for this purpose, we have

$$
\Gamma(\alpha+1)=\Gamma\left(\frac{2}{3}+1\right)=\Gamma\left(\frac{5}{3}\right)=0.89 .
$$

Thus,

$$
\frac{3 / 2 k}{\Gamma(\alpha+1)}=\frac{0.15}{0.89}=0.1685393<1 .
$$

Finally, all the conditions of Theorem 2 are satisfied; thus, we conclude that the intuitionistic fuzzy fractional boundary value problem (59) has a unique solution on $[0,1]$.

\section{Conclusion and Future Work}

In this paper, we establish the existence and uniqueness results of intuitionistic fuzzy local and nonlocal boundary value problems involving Caputo fractional derivative. The existence theorems are proved by using Banach fixed-point theorem and Schaefer's fixed-point theorem. As an 
application, an example is presented to illustrate the applicability of the obtained results.

Our future work is to study the existence and uniqueness results of intuitionistic fuzzy hybrid fractional differential equations [27-31].

\section{Data Availability}

The data used to support the findings of this study are included in the references within the article.

\section{Conflicts of Interest}

The authors declare that they have no conflicts of interest.

\section{References}

[1] R. P. Agarwal, V. Lakshmikantham, and J. J. Nieto, "On the concept of solution for fractional differential equations with uncertainty," Nonlinear Analysis, vol. 72, pp. 2859-2862, 2010.

[2] M. Arshad and S. Lupulescu, "On the fractional differential equations with uncertainty," Nonlinear Analysis, vol. 74, pp. 85-93, 2011.

[3] R. Alikhani and F. Bahrami, "Global solutions for nonlinear fuzzy fractional integral and integrodifferential equations," Communications in Nonlinear Science and Numerical Simulation, vol. 18, pp. 2007-2017, 2013.

[4] T. Allahviranloo, T. Gouyandeh, and Z. Armand, "Fuzzy fractional differential equations under generalized fuzzy Caputo derivative," Journal of Intelligent and Fuzzy Systems, vol. 26, pp. 1481-1490, 2014.

[5] S. Salahshour, T. Allahviranloo, T. Abbasbandy, and S. Baleanu, "Existence and uniqueness results for fractional differential equations with uncertainty," Advances in Difference Equations, vol. 1, pp. 1-12, 2012.

[6] N. V. Hoa, V. Lupulescu, and D. ORegan, "A note on initial value problems for fractional fuzzy differential equations," Fuzzy Sets and Systems, vol. 347, pp. 54-69, 2018.

[7] H. V. Ngo, V. Ho, and M. D. Tran, "Fuzzy fractional diferential equations under caputo-katugampola fractional derivative approach," Fuzzy Sets and Systems, vol. 375, pp. 70-99, 2018.

[8] V. Kumar and A. Alqahtani, "Lie symmetry analysis, soliton and numerical solutions of boundary value problem for variable coefficients coupled KdVBurgers equation," Nonlinear Dynamics, vol. 90, pp. 2903-2915, 2017.

[9] V. Kumar, L. Kumar, and M. E. Koksal, "Lie symmetry basedanalytical and numerical approach for modified Burgers-KdV equation," Results in physics, vol. 8, no. 8, pp. 1136-1142, 2018.

[10] A. Alqahtani and V. Kumar, "Soliton solutions to the timedependent coupled KdV-Burgers'equation," Advances in Difference Equations, vol. 1, pp. 1-16, 2019.

[11] M. Mazandarani and A. V. Kamyad, "Modified fractional Euler method for solving fuzzy fractional initial value problem," Communications in Nonlinear Science and $\mathrm{Nu}$ merical Simulation, vol. 18, pp. 12-21, 2013.

[12] H. V. Long, N. T. K. Son, and H. T. T. Tam, "The solvability of fuzzy fractional partial differential equations under caputo gH-differentiability," Fuzzy Sets and Systems, vol. 309, pp. 35-63, 2017.

[13] H. V. Long, "On random fuzzy fractional partial integrodifferential equations under Caputo generalized Hukuhara differentiability," Computational and Applied Mathematics, vol. 37, no. 3, pp. 2738-2765, 2018.

[14] K. Atanassov, "Intuitionistic fuzzy sets," Fuzzy Sets and Systems, vol. 20, pp. 87-96, 1986.

[15] L. A. Zadeh, "Fuzzy sets," Information and Control, vol. 8, no. 3, pp. 338-353, 1965.

[16] Z. S. Xu and R. Yager, "Some geometric aggregation operators based on intuitionistic fuzzy sets," International Journal of General Systems, vol. 35, pp. 417-433, 2006.

[17] S. Melliani, M. Elomari, L. S. Chadli, and R. Ettoussi, "Intuitionistic fuzzy fractional equation," Notes on Intuitionistic Fuzzy Sets, vol. 21, pp. 43-53, 2015.

[18] B. Bede, "Generalizations of the differentiability of fuzzynumber-valued functions with applications to fuzzy differential equations," Fuzzy Sets and Systems, vol. 151, pp. 581599, 2005

[19] S. Melliani, M. Elomari, L. S. Chadli, and R. Ettoussi, "Extension of Hukuhara difference in Intuitionistic fuzzy sets," Notes on Intuitionistic Fuzzy Sets, vol. 22, pp. 34-47, 2015.

[20] M. Elomari, S. Melliani, and L. S. Chadli, "Solution of intuitionistic fuzzy fractional differential equations," Annals of Fuzzy Mathematics and Informatics, vol. 201, pp. 22352287, 2016.

[21] Z. A. Smith, Fixed Point Methods in Nonlinear Analysis, The University of Chicago, Chicago, IL, USA, 2014, http://math. uchicago.edu/ may/REU2014/REUPapers/Smith,Z.pdf.

[22] J. Schauder, "Der fixpunktsatz in functionalraiumen," Studia Mathematica, vol. 2, pp. 171-180, 1930.

[23] N. Bourbaki, "General topology. Chapters 5-10, Translated from the French," in Elements of Mathematics, SpringerVerlag, Berlin, Germany, 1998.

[24] L. Byszewski, "Theorems about existence and uniqueness of solutions of a semilinear evolution nonlocal Cauchy problem," Journal of Mathematical Analysis and Applications, vol. 162, pp. 494-505, 1991.

[25] L. Byszewski, "Existence and uniqueness of mild and classical solutions of semilinear functional differential evolution nonlocal cauchy problem, selected problems of mathematics," Journal of Applied Mathematics and Stochastic Analysis, vol. 13, no. 2, 1995.

[26] L. Byszewski and V. Lakshmikantham, "Theorem about the existence and uniqueness of a solution of a nonlocal abstract cauchy problem in a banach space," Applicable Analysis, vol. 40, pp. 11-19, 1991.

[27] T. A. Burton and B. Zhang, "Fractional equations and generalizations of Schaefers and Krasnoselskiis fixed point theorems," Nonlinear Analysis, vol. 75, pp. 6485-6495, 2012.

[28] K. Diethelm and N. J. Ford, "Analysis of fractional differential equations," Journal of Mathematical Analysis and Applications, vol. 265, pp. 229-248, 2002.

[29] A. Kilbas, M. Srivastava, and J. Trujillo, "Theory and applications of fractional differential equations," North Holland Mathematical Studies, vol. 204, 2006.

[30] S. Momani and S. Hadid, "Lyapunov stability solutions of fractional integrodifferential equations," International Journal of Mathematics and Mathematical Sciences, vol. 47, pp. 2503-2507, 2004.

[31] J. Weissinger, "Zur theorie und anwendung des iterationsverfahrens," Mathematische Nachrichten, vol. 8, pp. 193-212, 1952. 